\title{
Predictive Factors of De Novo Overactive Bladder After Radical Prostatectomy in Patients With Clinically Localized Prostate Cancer: A Prospective Observational Study
}

\author{
Jun Nyung Lee, Sang Won Kim, Kyeong-Hyeon Byeon, Jae-Wook Chung, Yun-Sok Ha, \\ Seock Hwan Choi, Bum Soo Kim, Hyun Tae Kim, Tae-Hwan Kim, Eun Sang Yoo, Tae Gyun Kwon \\ Department of Urology, School of Medicine, Kyungpook National University, Daegu, Korea
}

\begin{abstract}
Purpose: To evaluate the incidence of de novo overactive bladder (OAB) and the factors related to its occurrence following radical prostatectomy (RP) in patients with clinically localized prostate cancer ( $\mathrm{PCa})$.

Materials and Methods: We prospectively examined 50 patients without OAB who underwent RP for clinically localized PCa in our institution from August 2019 to February 2020. We performed assessments using the International Prostate Symptom Score (IPSS), the Overactive Bladder Symptom Score (OABSS), and uroflowmetry before surgery and 3 months after RP. OAB was defined as a score of 1 or more on the urgency components of the OABSS. Three months after RP, the patients were divided into 2 groups based on the presence of de novo OAB symptoms. We evaluated the patients' demographics and outcomes after RP according to their de novo OAB grouping. The predictive factors of de novo OAB after RP were analyzed using a multivariate logistic regression model.

Results: Of the 50 patients, 22 (44\%) had de novo OAB 3 months after RP. The patients in the de novo OAB group were older, had higher preoperative IPSS storage subscores, and had larger volumes of postvoid residual urine on preoperative uroflowmetry than those in the non-de novo OAB group. Multivariate analysis showed that age and preoperative IPSS storage subscores were predictive factors of de novo OAB after RP.

Conclusions: De novo OAB was observed in $44 \%$ of the patients 3 months after RP. Age and preoperative IPSS storage subscores were predictive factors of de novo OAB following RP.
\end{abstract}

Key Words: Prostate cancer, Prostatectomy, Overactive urinary bladder
Received January 12, 2021

Revised January 22, 2021

Accepted January 26, 2021

Corresponding author:

Tae Gyun Kwon

Email: tgkwon@knu.ac.kr https://orcid.org/0000-0002-4390-0952

This work was supported by Biomedical Research Institute grant, Kyungpook National University Hospital (2019).

\section{INTRODUCTION}

Prostate cancer $(\mathrm{PCa})$ is a common cancer in men, and its incidence is rapidly increasing. ${ }^{1}$ Although radical prostatectomy $(R P)$ is considered the definitive standard treatment for clinically localized $\mathrm{PCa},{ }^{2}$ it can cause surgical complications, including urinary incontinence and erectile dysfunction, adversely affecting the patients' quality of life
(QoL). ${ }^{3}$ Postprostatectomy incontinence is an especially well-recognized complication with a negative impact on the QoL. ${ }^{4}$

Urinary incontinence is classified into stress urinary incontinence (SUI) and urge urinary incontinence (UUI); however, most studies on postprostatectomy incontinence have focused on SUI. Although several studies on SUI after RP were conducted,,$^{5-7}$ patients can present with various 
lower urinary tract symptoms (LUTS) following a radical surgery. ${ }^{8}$ Although the deterioration of urinary storage symptoms such as de novo overactive bladder (OAB) is expected after RP, the prevalence of and predisposing factors of the development of urinary storage symptoms after RP are not well known. Recently, studies have shown the prevalence of de novo $\mathrm{OAB}$ and its negative impact on the QoL in men following treatment for localized PCa. ${ }^{9-13}$ In this study, we investigated the incidence of de novo $\mathrm{OAB}$ and the predisposing factors related to its occurrence after RP in patients with clinically localized PCa.

\section{MATERIALS AND METHODS}

\section{Study Participants}

This study was approved by the Institutional Review Board (IRB) of Kyungpook National University Chilgok Hospital (IRB NO. KNUCH 201906-007). Between August 2019 and February 2020, 113 patients underwent robot-assisted RP (RARP) and 32 patients underwent open RP (ORP) for PCa at our institution. Of them, we prospectively enrolled 50 patients without preoperative OAB. We included patients with clinically localized PCa (clinical stage $\leq$ T2cNOM0) and urgency subscore (Q3)/UUI subscore $(\mathrm{Q})=0 / 0$ on the preoperative Overactive Bladder Symptom Score (OABSS) questionnaire. We excluded patients who had a history of receiving hormonal therapy or radiotherapy before RP; medical or surgical treatment for OAB; neurological disorders such as stroke, Parkinson disease, and multiple sclerosis; spinal cord injuries (neurogenic bladder dysfunction); bladder calculi; active urinary tract infections; severe cardiac diseases; renal dysfunctions; and hepatic dysfunctions. In addition, we excluded those without a followup period of $>3$ months from the study. During the study period, we did not prescribe $O A B$ medications including muscarinic antagonists and $\beta 3$ agonists to the patients included in the study during the 3-month period after RP.

\section{Surgical Procedures}

A single surgeon performed all operations. The RARP and ORP procedures were conducted via a transperitoneal approach using the da Vinci $\mathrm{Xi}$ or $\mathrm{Si}$ system and a retropubic approach, respectively. Previously, we reported a modified technique for RARP and ORP at our institution. ${ }^{14}$ Briefly, the bladder neck was dissected first. After the dissection of the vas deferens and seminal vesicle, the prostate was dissected in an antegrade fashion from the bladder neck with nerve sparing depending on the patient's cancer status. Finally, the urethra was cut at the prostate apex. After a Rocco suture ${ }^{15}$ was applied, urethrovesical anastomosis was performed using continuous sutures. The urethral catheter was removed on postoperative day 6 .

\section{Symptom Scoring}

In this study, the International Prostate Symptom Score (IPSS), the OABSS, and uroflowmetry were assessed before surgery (within 1 month preoperatively) and 3 months after surgery. In addition, the total scores and the voiding, and storage symptom subscores of the IPSS were evaluated. A postvoid residual volume (PVR) greater than $50 \mathrm{~mL}$ was considered significant as a categorized variable. De novo OAB was defined as a score of one or more on the urgency components of the OABSS 3 months after surgery. Additionally, the absence of stress incontinence (involuntary leakage of urine with exertion) was defined as the use of zero pads per day. We evaluated the incidence of de novo $\mathrm{OAB}$ after dividing the patients into 2 groups (the de novo $\mathrm{OAB}$ and non-de novo OAB groups) 
based on the presence of de novo OAB symptoms 3 months after RP. We compared the patients' demographics and outcomes after RP according to their de novo OAB grouping.

\section{Statistical Analysis}

We assessed the associations between variables in the 2 groups using Student t-test and the chisquare test. A multivariate logistic regression analysis was conducted to identify the predictive factors of de novo OAB in patients who underwent RP. The statistical analyses were performed using IBM SPSS Statistics ver. 23.0 (IBM Co., Armonk, NY, USA), and $p$-values of $<0.05$ were used to denote statistical significance.

\section{RESULTS}

Fifty patients without preoperative $\mathrm{OAB}$ who underwent $\mathrm{RP}$ were included in this study. De novo OAB was observed in 22 patients (44.0\%) 3 months after RP, while 28 patients (56.0\%) did not have any de novo OAB symptoms after RP. Preoperative patient demographics between the 2 groups are presented in Table 1 . The mean age of the patients was 64.9 years. The patients in the de novo OAB group were significantly older than those in the non-de novo OAB group (67.4 \pm 5.5 years vs. $63.1 \pm$ 6.7 years, respectively, $\mathrm{p}=0.018$ ). Although stresstype urinary incontinence was observed in $16 \%$ (8 of 50) of the patients 3 months after RP, there was no significant difference between the groups. The prostate size, preoperative prostate-specific

Table 1. Comparison of the clinical and pathological characteristics according to de novo overactive bladder after radical prostatectomy

\begin{tabular}{|c|c|c|c|c|}
\hline Characteristic & Total patients $(n=50)$ & De novo OAB $(\mathrm{n}=22)$ & Non-de novo OAB $(\mathrm{n}=28)$ & $\mathrm{p}$-value \\
\hline Age (yr) & $64.9 \pm 6.5$ & $67.4 \pm 5.5$ & $63.1 \pm 6.7$ & 0.018 \\
\hline Prostate size $(\mathrm{mL})$ & $30.1 \pm 9.9$ & $31.7 \pm 12.8$ & $28.8 \pm 7.0$ & 0.353 \\
\hline $\operatorname{BMI}\left(\mathrm{kg} / \mathrm{m}^{2}\right)$ & $23.7 \pm 3.0$ & $23.9 \pm 2.2$ & $23.5 \pm 3.6$ & 0.646 \\
\hline PSA (ng/mL) & $8.7 \pm 8.2$ & $7.7 \pm 4.3$ & $9.6 \pm 10.3$ & 0.425 \\
\hline IIEF-5 & $11.4 \pm 6.7$ & $10.2 \pm 6.8$ & $12.4 \pm 6.5$ & 0.240 \\
\hline Drinking, $-/+$ & $39 / 11$ & $17 / 5$ & $22 / 6$ & 0.912 \\
\hline Smoking, $-/+$ & $34 / 16$ & $18 / 4$ & $16 / 12$ & 0.063 \\
\hline HTN, $-/+$ & $31 / 19$ & $13 / 9$ & $18 / 10$ & 0.707 \\
\hline DM, -/+ & $34 / 16$ & $15 / 7$ & $19 / 9$ & 0.981 \\
\hline Biopsy GS, 6/7/8/9 & $21 / 21 / 6 / 2$ & $9 / 7 / 4 / 2$ & $12 / 14 / 2 / 0$ & 0.190 \\
\hline Total positive core number & $2.8 \pm 1.5$ & $2.7 \pm 1.9$ & $2.9 \pm 1.4$ & 0.615 \\
\hline Positive core number, $<3 / \geq 3$ & $26 / 24$ & $10 / 12$ & $16 / 12$ & 0.412 \\
\hline Clinical T stage, $\mathrm{T} 1 \mathrm{c} / \mathrm{T} 2$ & $22 / 28$ & $9 / 13$ & $13 / 15$ & 0.696 \\
\hline D'Amico group & & & & 0.172 \\
\hline Low & 17 & 7 & 10 & \\
\hline Intermediate & 25 & 9 & 16 & \\
\hline High & 8 & 6 & 2 & \\
\hline Operative methods, robot/open & $42 / 8$ & $20 / 2$ & $22 / 6$ & 0.439 \\
\hline Nerve sparing, uni-/bilateral & $13 / 37$ & $6 / 16$ & $7 / 21$ & 0.856 \\
\hline Operative time ( $\mathrm{min})$ & $131.7 \pm 35.0$ & $135.9 \pm 24.6$ & $128.4 \pm 40.8$ & 0.426 \\
\hline Pathologic T stage, T2/T3 & $31 / 19$ & $13 / 9$ & $18 / 10$ & 0.707 \\
\hline Pathologic GS, 6/7/8/9 & $18 / 19 / 11 / 2$ & $8 / 7 / 5 / 2$ & $10 / 12 / 6 / 0$ & 0.478 \\
\hline Positive surgical margin, $-/+$ & $38 / 12$ & $17 / 5$ & $21 / 7$ & 0.852 \\
\hline Stress incontinence, $-/+$ & $42 / 8$ & $16 / 6$ & $26 / 2$ & 0.116 \\
\hline
\end{tabular}

Values are presented as mean \pm standard deviation or number.

OAB: overactive bladder, BMI: body mass index, PSA: prostate-specific antigen, IIEF: International Index of Erectile Function, HTN: hypertension, DM: diabetes mellitus, GS: Gleason score. 
antigen, D'amico risk classification, operative methods (robot vs. open), degree of nerve sparing, operative time, pathological $\mathrm{T}$ stage, pathological grade, and margin status were not significantly different between the 2 groups.

Table 2 shows the IPSS, OABSS, and uroflowmetry variables before surgery and 3 months after surgery. The preoperative IPSS storage symptom score was significantly higher in the de novo $\mathrm{OAB}$ group than that in the non-de novo OAB group $(5.3 \pm 2.9$ and $2.9 \pm 1.7$, respectively, $\mathrm{p}=0.002$ ). Although the total IPSS score was also higher in the de novo OAB group than that in the non-de novo OAB group, this difference was not statistically significant (10.6 \pm 8.8 and 6.9 \pm 5.8 , respectively, $p=0.074$ ). Preoperatively, the patients in the de novo $\mathrm{OAB}$ group demonstrated a higher prevalence of PVR of $>50 \mathrm{~mL}$ than those in the non-de novo $\mathrm{OAB}$ group $(36.4 \%$ and $7.1 \%$, respectively, $\mathrm{p}=0.014)$. The preoperative IPSS voiding symptom score, OABSS, maximal flow rate, and voided volume did not show any significant differences between the groups.

Multivariate analysis showed that age and preoperative IPSS storage symptom score were significant factors related to the development of de novo $\mathrm{OAB}$ after RP (odds ratio [OR], 1.169; 95\% confidence interval [CI], 1.020-1.339; $\mathrm{p}=0.025$ and OR, 1.690; 95\% CI, 1.180-2.422; p=0.004, respectively) (Table 3 ). Receiver operating curve analysis determined that the optimal cutoff value for age was 64.5 years old (sensitivity, $72.7 \%$; specificity, $57.1 \%$ ) and that for the preoperative IPSS storage symptom score was 3.5 (sensitivity, 72.7\%; specificity, 64.3\%).

\section{DISCUSSION}

Although various LUTSs may occur after $\mathrm{RP},{ }^{8}$ many studies have only focused on stress-type urinary incontinence. ${ }^{5-7}$ Therefore, we assessed the incidence of de novo $\mathrm{OAB}$ and the significant factors related to its occurrence after RP in patients with clinically localized PCa. In this study,

Table 3. Multivariable logistic regression analyses of the predictive factors associated with de novo overactive bladder after radical prostatectomy

\begin{tabular}{lcc}
\hline \multicolumn{1}{c}{ Variable } & OR $(95 \% \mathrm{CI})$ & p-value \\
\hline Age $(\mathrm{yr})$ & $1.169(1.020-1.339)$ & 0.025 \\
IPSS storage symptom score & $1.690(1.180-2.422)$ & 0.004 \\
PVR (mL) & $1.011(1.003-1.020)$ & 0.445 \\
\hline
\end{tabular}

OR: odds ratio, CI: confidence interval, IPSS: International Prostate Symptom Score, PVR: postvoid residual volume.

Table 2. Comparison of the preoperative questionnaire and uroflowmetry according to de novo overactive bladder after radical prostatectomy

\begin{tabular}{|c|c|c|c|c|}
\hline Variable & Total patients $(n=50)$ & De novo OAB $(\mathrm{n}=22)$ & Non-de novo OAB ( $\mathrm{n}=28)$ & p-value \\
\hline IPSS total score & $8.5 \pm 7.4$ & $10.6 \pm 8.8$ & $6.9 \pm 5.8$ & 0.074 \\
\hline IPSS voiding symptom score & $4.6 \pm 5.8$ & $5.4 \pm 6.8$ & $4.0 \pm 4.9$ & 0.415 \\
\hline IPSS storage symptom score & $3.9 \pm 2.6$ & $5.3 \pm 2.9$ & $2.9 \pm 1.7$ & 0.002 \\
\hline OABSS & $1.9 \pm 0.8$ & $1.8 \pm 0.8$ & $1.9 \pm 0.9$ & 0.662 \\
\hline \multicolumn{5}{|l|}{ UFM } \\
\hline Maximal flow rate $(\mathrm{mL} / \mathrm{sec})$ & $12.7 \pm 5.3$ & $13.1 \pm 6.2$ & $12.4 \pm 4.6$ & 0.633 \\
\hline $\mathrm{VV}(\mathrm{mL})$ & $224.1 \pm 110.4$ & $245.5 \pm 130.6$ & $207.3 \pm 90.4$ & 0.228 \\
\hline PVR (mL) & & & & 0.014 \\
\hline$\leq 50$ & 40 & 14 & 26 & \\
\hline$>50$ & 10 & 8 & 2 & \\
\hline
\end{tabular}

OAB: overactive bladder, IPSS: International Prostate Symptom Score, OABSS: overactive bladder symptom score, UFM: uroflowmetry, VV: voided volume, PVR: postvoid residual volume. 
we observed a high prevalence (44.0\%) of de novo OAB 3 months after RP. In addition, multivariate logistic regression analysis showed that both age and preoperative IPSS storage symptom scores were significant factors related to the development of de novo OAB following RP.

Even though studies, including the ProtecT trial, revealed that men with $\mathrm{PCa}$ have similar LUTS to control patients at baseline, ${ }^{16}$ following PCa therapy, they can present with various LUTS. ${ }^{8}$ In addition, RP is associated with the considerable deterioration of LUTSs compared to other treatment strategies such as active surveillance, radiotherapy, and hormone therapy, despite the high survival rate after RP. ${ }^{17,18}$ Therefore, in addition to the eradication of $\mathrm{PCa}$ itself, it is also important to recognize and control the nuisance and discomfort of postoperative LUTSs in patients. The epidemiology, pathophysiology, and treatment of SUI after RP have been investigated in detail, and the main etiological factor of SUI is the dysfunction of the urethral sphincter. ${ }^{5-7}$ Although urinary storage symptoms are reported to be more bothersome and more likely to reduce the QoL than voiding and postmicturition symptoms, ${ }^{19,20}$ few studies have described and clarified the causes of urinary storage dysfunctions such as UUI and de novo OAB after RP. Because many patients undergo RP for clinically localized PCa globally, it is meaningful to investigate the prevalence and related factors of de novo $\mathrm{OAB}$ after RP.

$O A B$ negatively impacts the QoL and often requires medications with troublesome side effects. ${ }^{21}$ A recent survey study found that $11 \%$ and $30 \%$ of the patients treated with RP and radiotherapy, respectively, had OAB 36 months after therapy. ${ }^{9}$ The rates of de novo urodynamic detrusor overactivity following RP historically were approximately $2 \%-77 \%$ and tended to persist in most patients (83\%). ${ }^{22} \mathrm{~A}$ contemporary literature review reported similar findings, with $2 \%-63 \%$ of the patients studied developing urodynamic detrusor overactivity following RP. ${ }^{18}$ A recent large cohort retrospective study involving 875 men who underwent ORP has reported that the incidence of de novo OAB was $19 \%$, with $29 \%$ of the patients developing one or more urinary storage symptoms and $6 \%$ reporting UUI at a median follow-up of 2.7 years after RP. ${ }^{11}$ The authors suggested that adjuvant or salvage radiotherapy was associated with a significant increase in the risk of developing OAB. Furthermore, OAB symptoms progressively developed during their retrospective study, and less than half of patients received $O A B$ treatment. ${ }^{11}$ In the present prospective study, de novo OAB was observed in $44 \%$ of the patients without preoperative OAB 3 months after RP. Although this prospective study showed a higher prevalence of de novo OAB than other studies possibly due to its patient inclusion criteria and strict assessment method and timing, our results provided evidence that $\mathrm{OAB}$ and storage LUTSs are common after RP.

Several studies have shown that recovery from urinary continence after RP is related to several patient- or surgery-related factors including age, ${ }^{21,23,24}$ body mass index, ${ }^{25}$ prostate volume, ${ }^{24,25}$ preoperative IPSS score, ${ }^{23}$ the length of the membranous urethra, ${ }^{26}$ and nerve preservation. ${ }^{27}$ However, the predisposing factors of the development of urinary storage symptoms after RP remain relatively unknown. Recently, Matsukawa et al. ${ }^{12}$ have reported in an RARP series a similar incidence of de novo OAB to the present study. They showed that preoperative IPSS-QoL scores and postoperative maximum urethral closing pressure in a urodynamic study were significant predictive factors of de novo $\mathrm{OAB}$ in a multivariable logistic analysis. In addition, Yamada et al. ${ }^{13}$ investigated the clinical significance of preoperative urinary storage symptoms on the continence recovery 12 months 
after RARP. They revealed that the presence of preoperative $\mathrm{OAB}$ was an independent negative predictor of continence 12 months after RARP, and both the diagnosis and severity of $O A B$ were associated with continence recovery. Furthermore, they suggested that patients with $O A B$ are more vulnerable to developing incontinence after RARP. Similarly, our study showed that preoperative IPSS storage symptom score was a predictor of de novo $\mathrm{OAB}$ after RP in patients without preoperative $\mathrm{OAB}$. We suggest that de novo OAB after RP occurs postoperatively in patients with preoperative urinary storage dysfunction, even without preoperative $\mathrm{OAB}$. In this study, multivariate analysis showed that age was also a predictive factor of de novo OAB after surgery. Studies have indicated a positive correlation between age and $\mathrm{OAB}$ in the general population. ${ }^{28,29}$ Researchers assumed that age-related changes in the bladder and pelvic floor tissues including the nervous system might contribute to the high occurrence of OAB in elderly patients. Therefore, physicians should not overlook the occurrence of de novo $\mathrm{OAB}$ and its predisposing factors after surgery in patients with clinically localized PCa. Although our study did not conduct a urodynamic study, age and preoperative IPSS storage symptom score were valuable because performing an invasive study in most patients undergoing RP is difficult.

Although the mechanism of the development of de novo urinary storage dysfunctions after RP is still incompletely understood, we assumed that the mechanisms could be related to the neural and anatomical disruptions at the surgical site, which can lead to bladder denervation. Traction injury to the bladder during surgery may contribute to reduced bladder perfusion and later neural and detrusor dysfunction. Porena et al. ${ }^{22}$ compared preoperative and postoperative urodynamics after surgery and found reduced compliance in 8\%-39\% of patients, half of whom had new symptoms commonly associated with other voiding problems (such as SUI and detrusor overactivity). This finding persisted in $28 \%$ of the patients for 3 years. ${ }^{30}$ In addition, prolonged high intravesical pressures preoperatively may ultimately contribute to reduced bladder perfusion and later neural and detrusor dysfunction. The preoperative IPSS storage symptom score identified as a predictive factor in this study could be considered a representative feature of reduced bladder compliance.

There were several limitations to consider in this study. First, this study did not investigate the long-term outcomes of LUTSs including de novo $\mathrm{OAB}$ after RP. The high prevalence of de novo $\mathrm{OAB}$ in this study might be overestimated because the assessment timing was made at the very early period after surgery. In addition, a small number of patients were included in this prospective study because of the strict inclusion criteria. Moreover, de novo $\mathrm{OAB}$ was diagnosed using a questionnaire completed by the patients, and a urodynamic study including measurement values of the bladder and urethra were not conducted. These limitations warrant further long-term prospective studies including urodynamics with a larger number of patients to clarify the natural history and mechanism of the development of LUTSs after RP in patients with PCa.

\section{CONCLUSIONS}

In this study, we observed a considerable incidence rate (44\%) of de novo OAB 3 months after RP in patients with clinically localized PCa. In addition, age and preoperative IPSS storage symptom scores were predictive factors of de novo OAB following RP. Our results suggest that physicians should consider the occurrence and predictive factors of de novo OAB after RP in patients with clinically localized PCa. 


\section{CONFLICT OF INTEREST}

The authors claim no conflicts of interest.

\section{REFERENCES}

1. Han HH, Park JW, Na JC, Chung BH, Kim CS, Ko WJ. Epidemiology of prostate cancer in South Korea. Prostate Int 2015;3:99-102.

2. Nepple KG, Stephenson AJ, Kallogjeri D, Michalski J, Grubb RL 3rd, Strope SA, et al. Mortality after prostate cancer treatment with radical prostatectomy, external-beam radiation therapy, or brachytherapy in men without comorbidity. Eur Urol 2013;64:3728.

3. Frank SJ, Pisters LL, Davis J, Lee AK, Bassett R, Kuban DA. An assessment of quality of life following radical prostatectomy, high dose external beam radiation therapy and brachytherapy iodine implantation as monotherapies for localized prostate cancer. J Urol 2007;177:2151-6.

4. Sanda MG, Dunn RL, Michalski J, Sandler HM, Northouse L, Hembroff L, et al. Quality of life and satisfaction with outcome among prostate-cancer survivors. N Engl J Med 2008;358:1250-61.

5. Dubbelman YD, Groen J, Wildhagen MF, Rikken B, Bosch JLHR. Urodynamic quantification of decrease in sphincter function after radical prostatectomy: relation to postoperative continence status and the effect of intensive pelvic floor muscle exercises. Neurourol Urodyn 2012;31:646-51.

6. Matsukawa Y, Hattori R, Yoshikawa Y, Ono Y, Gotoh M. Laparoscopic versus open radical prostatectomy: urodynamic evaluation of vesicourethral function. Int J Urol 2009;16:393-6.

7. Kadono Y, Ueno S, Kadomoto S, Iwamoto H, Takezawa Y, Nakashima K, et al. Use of preoperative factors including urodynamic evaluations and nerve-sparing status for predicting urinary continence recovery after robot-assisted radical prostatectomy: nerve-sparing technique contributes to the reduction of postprostatectomy incontinence. Neurourol Urodyn 2016;35:1034-9.

8. Biers S, Sievert KD, Thiruchelvam N. Overactive bladder syndrome and lower urinary tract symptoms after prostate cancer treatment. Curr Opin Urol 2017;27:307-13.

9. Boettcher M, Haselhuhn A, Jakse G, Brehmer B, Kirschner-Hermanns R. Overactive bladder syndrome: an underestimated long-term problem after treatment of patients with localized prostate cancer? BJU Int 2012;109:1824-30.

10. Thiruchelvam N, Cruz F, Kirby M, Tubaro A, Chapple CR, Sievert KD. A review of detrusor overactivity and the overactive bladder after radical prostate cancer treatment. BJU Int 2015;116:853-61.

11. Hosier GW, Tennankore KK, Himmelman JG, Gajewski J, Cox AR. Overactive bladder and storage lower urinary tract symptoms following radical prostatectomy. Urology 2016;94:193-7.

12. Matsukawa Y, Yoshino Y, Ishida S, Fujita T, Majima T, Funahashi $Y$, et al. De novo overactive bladder after robot-assisted laparoscopic radical prostatectomy. Neurourol Urodyn 2018;37:2008-14.

13. Yamada Y, Fujimura T, Fukuhara H, Sugihara T, Miyazaki H, Nakagawa T, et al. Overactive bladder is a negative predictor of achieving continence after robot-assisted radical prostatectomy. Int J Urol 2017;24:749-56.

14. Kwon SY, Lee JN, Ha YS, Choi SH, Kim TH, Kwon TG. Open radical prostatectomy reproducing robot-assisted radical prostatectomy: involving antegrade nerve sparing and continuous anastomosis. Int Braz J Urol 2017;43:1043-51.

15. Rocco F, Rocco B. Anatomical reconstruction of the rhabdosphincter after radical prostatectomy. BJU Int 2009;104:274-81.

16. Lane A, Metcalfe C, Young GJ, Peters TJ, Blazeby $\mathrm{J}$, Avery KN, et al. Patient-reported outcomes in the ProtecT randomized trial of clinically localized prostate cancer treatments: study design, and baseline urinary, bowel and sexual function and quality 
of life. BJU Int 2016;118:869-79.

17. Donovan JL, Hamdy FC, Lane JA, Mason M, Metcalfe C, Walsh E, et al. Patient-reported outcomes after monitoring, surgery, or radiotherapy for prostate cancer. N Engl J Med 2016;375:1425-37.

18. Hamdy FC, Donovan JL, Lane JA, Mason M, Metcalfe C, Holding P, et al. 10-year outcomes after monitoring, surgery, or radiotherapy for localized prostate cancer. N Engl J Med 2016;375:1415-24.

19. Agarwal A, Eryuzlu LN, Cartwright R, Thorlund K, Tammela TLJ, Guyatt GH, et al. What is the most bothersome lower urinary tract symptom? Individual- and population-level perspectives for both men and women. Eur Urol 2014;65:1211-7.

20. Irwin DE, Milsom I, Hunskaar S, Reilly K, Kopp Z, Herschorn S, et al. Population-based survey of urinary incontinence, overactive bladder, and other lower urinary tract symptoms in five countries: results of the EPIC study. Eur Urol 2006;50:1306-14.

21. Milsom I, Kaplan SA, Coyne KS, Sexton CC, Kopp ZS. Effect of bothersome overactive bladder symptoms on health-related quality of life, anxiety, depression, and treatment seeking in the United States: results from EpiLUTS. Urology 2012;80:90-6.

22. Porena M, Mearini E, Mearini L, Vianello A, Giannantoni A. Voiding dysfunction after radical retropubic prostatectomy: more than external urethral sphincter deficiency. Eur Urol 2007;52:38-45.

23. Lavigueur-Blouin H, Noriega AC, Valdivieso R, Hueber PA, Bienz M, Alhathal N, et al. Predictors of early continence following robot-assisted radical prostatectomy. Can Urol Assoc J 2015;9:e93-7.

24. Kim JJ, Ha YS, Kim JH, Jeon SS, Lee DH, Kim WJ, et al. Independent predictors of recovery of continence 3 months after robot-assisted laparoscopic radical prostatectomy. J Endourol 2012;26:1290-5.

25. Boczko J, Erturk E, Golijanin D, Madeb R, Patel H, Joseph JV. Impact of prostate size in robot-assisted radical prostatectomy. J Endourol 2007;21:184-8.

26. Mungovan SF, Sandhu JS, Akin O, Smart NA, Graham PL, Patel MI. Preoperative membranous urethral length measurement and continence recovery following radical prostatectomy: a systematic review and meta-analysis. Eur Urol 2017;71:368-78.

27. Ludovico GM, Dachille G, Pagliarulo G, D’Elia C, Mondaini N, Gacci M, et al. Bilateral nerve sparing robotic-assisted radical prostatectomy is associated with faster continence recovery but not with erectile function recovery compared with retropubic open prostatectomy: the need for accurate selection of patients. Oncol Rep 2013;29:2445-50.

28. Zhu JZ, Hu XY, Dong XY, Li LK. Associations between risk factors and overactive bladder: a metaanalysis. Female Pelvic Med Re 2019;25:238-46.

29. Kim SY, Bang W, Choi HG. Analysis of the prevalence and associated factors of overactive bladder in adult Korean men. PLoS One 2017;12:e0175641.

30. Giannantoni A, Mearini E, Zucchi A, Costantini E, Mearini L, Bini V, et al. Bladder and urethral sphincter function after radical retropubic prostatectomy: a prospective long-term study. Eur Urol 2008;54:657-64. 